\title{
ON THE MULTIPLICITIES OF THE POWERS OF A BANACH SPACE OPERATOR
}

\author{
DOMINGO A. HERRERO $\sigma^{1}$
}

\begin{abstract}
The multiplicities of the powers of a bounded linear operator $T$, acting on a complex separable infinite-dimensional Banach space $\mathscr{X}$, satisfy the inequalities

$(* *) \quad \mu\left(T^{n}\right) \leqslant \mu\left(T^{h n}\right) \leqslant h \mu\left(T^{n}\right)$ for all $h, n \geqslant 1$.

Nothing else can be said, in general, because simple examples show that for each sequence $\left\{\mu_{n}\right\}_{n=1}^{\infty}$, satisfying the inequalities $(* *)$, there exists $T$ acting on $\mathscr{X}$ such that $\mu\left(T^{n}\right)=\mu_{n}$ for all $n \geqslant 1$.
\end{abstract}

Let $\mathscr{L}(\mathscr{X})$ denote the algebra of all (bounded linear) operators acting on a complex separable infinite-dimensional Banach space $\mathscr{X}$. The multiplicity of $T$ in $\mathscr{L}(\mathscr{X})$ is the cardinal number defined by

$$
\mu(T)=\min _{\Gamma \subset \mathscr{X}}\left\{\mathbf{c}(\Gamma): \mathscr{X}=\bigvee\left\{T^{n} y: y \in \Gamma, n=0,1,2, \ldots\right\}\right\},
$$

where $\bigvee \mathscr{R}$ denotes the closed linear span of the vectors in $\mathscr{R}$. It is immediate from the definition that

$$
\mu\left(T^{n}\right) \leqslant \mu\left(T^{h n}\right) \leqslant h \mu\left(T^{n}\right) \text { for all } h, n \geqslant 1 .
$$

Thus, in particular, if $T^{n}$ is cyclic (i.e., $\mu\left(T^{n}\right)=1$ ) for some $n \geqslant 1$, then $T^{m}$ is also cyclic for all $m \mid n$ (where $m \mid n$ indicates that $m$ divides $n$ ).

This is the only possible general result relating the multiplicities of the different powers of a given operator. Indeed, we have the following result.

THEOREM 1. Let $\mathscr{X}$ be a complex separable infinite-dimensional Banach space. Given a sequence $M=\left\{\mu_{n}\right\}_{n=1}^{\infty}$ of natural numbers, satisfying the inequalities

$$
\mu_{n} \leqslant \mu_{h n} \leqslant h \mu_{n} \text { for all } h, n \geqslant 1 \text {, }
$$

there exists a nuclear operator $T(M)$ in $\mathscr{L}(\mathscr{X})$ such that

$$
\mu\left(T(M)^{n}\right)=\mu_{n} \text { for all } n=1,2, \ldots
$$

For the Hilbert space case, we have

THEOREM 2. Let $\mathscr{H}$ be a complex separable infinite-dimensional Hilbert space. Given a sequence $M$ satisfying the conditions of Theorem 1, there exists a normal operator $N(M)$ in $\mathscr{L}(\mathscr{H})$ such that

$$
\mu\left(N(M)^{n}\right)=\mu_{n} \text { for all } n=1,2, \ldots, \text { and } \sigma(N(M))=\{\lambda:|\lambda| \leqslant 1\},
$$
where $\sigma(N(M))$ denotes the spectrum of $N(M)$.

Received by the editors February 27, 1984 and, in revised form, July 6, 1984

1980 Mathematics Subject Classification. Primary 47A99, 47B10.

Key words and phrases. Multiplicity of an operator, powers.

${ }^{1}$ This research was partially supported by a grant from the National Science Foundation 
Let $\left\{e_{i}\right\}_{i=1}^{\mu_{n}}$ be the canonical orthonormal basis of $\mathbf{C}^{\mu_{n}}$ and let $e(t)=\exp \{2 \pi i t\}(t$ a real number). If $\alpha_{0}+\alpha_{1}+\alpha_{2}+\cdots+\alpha_{n-1}=\mu_{n}$, then we write

$$
A=A\left(\alpha_{0}, \alpha_{1}, \alpha_{2}, \ldots, \alpha_{n-1}\right)=\bigoplus_{j=0}^{n-1} e\left(\frac{j}{n}\right)^{\left(\alpha_{j}\right)}
$$

to indicate the diagonal (and therefore normal) operator defined by

$$
A e_{i}= \begin{cases}e_{i}, \quad 1 \leqslant i \leqslant & \alpha_{0}, \\ e(j / n) e_{i}, & \alpha_{0}+\alpha_{1}+\cdots+\alpha_{j-1}<i \leqslant \alpha_{0}+\alpha_{1}+\cdots+\alpha_{j} \\ & (j=1,2, \ldots, n-1) .\end{cases}
$$

Observe that $A^{n}$ is the identity operator, and therefore

$$
\mu\left(A^{n}\right)=\operatorname{dim} \mathbf{C}^{\mu_{n}}=\mu_{n} .
$$

It is clear that every operator satisfying this condition must also satisfy

$$
\begin{aligned}
\mu\left(A^{m}\right) & \geqslant\left\{\text { smallest integer greater than or equal to } m \mu_{n} / n\right\} \\
& =\left[\left(m \mu_{n}+n-1\right) / n\right]=\left[\left(m \mu_{n}-1\right) / n\right]+1
\end{aligned}
$$

for each $m \mid n$, where $[t]$ denotes the integral part of the real number $t$. (To see this, use (1).)

Observe that, for each $k \geqslant 1$,

$$
\mu\left(A^{k}\right)=\mu\left(\bigoplus_{j=0}^{n-1} e\left(\frac{k j}{n}\right)^{\left(\alpha_{j}\right)}\right)=\max _{0 \leqslant t<n} \sum\left\{\alpha_{j}: k j \equiv t(\bmod n)\right\}
$$

(with the convention that $\sum\left\{\alpha_{j}: k j \equiv t(\bmod n)\right\}=0$ if $k j \not \equiv t(\bmod n)$ for all $j=0,1,2, \ldots, n-1)$.

The key result is Lemma 3 below, which says that, for a clever choice of $\alpha_{0}, \alpha_{1}, \ldots, \alpha_{n-1}, \mu\left(A^{k}\right)$ does not exceed $\mu\left(B^{k}\right)$ for any operator $B$ such that $\mu\left(B^{n}\right)=\mu_{n}($ for all $k=1,2, \ldots)$.

LEMMA 3. Let $n, \mu_{n} \geqslant 1$ and let

$$
s_{n}=\left[\left(\mu_{n}-1\right) / n\right]+1 \text { and } a_{n}=\mu_{n}-n\left(s_{n}-1\right) \text {. }
$$

If

$$
A_{n}=\left\{\bigoplus_{j=0}^{a_{n}-1} e\left(\frac{j}{n}\right)^{\left(s_{n}\right)}\right\} \oplus\left\{\bigoplus_{j=a_{n}}^{n-1} e\left(\frac{j}{n}\right)^{\left(s_{n}-1\right)}\right\}
$$

then $\mu\left(A_{n}^{n}\right)=\mu_{n}$ and

$$
\begin{aligned}
\mu\left(A_{n}^{k}\right) & =\mu\left(A_{n}^{(k, n)}\right)=\left[\left((k, n) \mu_{n}-1\right) / n\right]+1 \\
& =\min \left\{\mu\left(B^{k}\right): B \in \mathscr{L}(\mathscr{X}), \mu\left(B^{n}\right)=\mu_{n}\right\}
\end{aligned}
$$

for all $k=1,2, \ldots$, where $(k, n)=$ G.C.D. $\{k, n\}$ and $\mathscr{X}$ is an arbitrary complex separable Banach space with $\operatorname{dim} \mathscr{X} \geqslant \mu_{n}$. 
Proof. It follows from (3) and (5) that

$$
\mu\left(A_{n}^{n}\right)=\mu\left(A_{n}^{h n}\right)=\mu_{n} \quad(h=1,2, \ldots)
$$

and

$$
\mu\left(A_{n}\right)=\max \left\{s_{n}, s_{n}-1\right\}=s_{n} .
$$

Let $\mathbf{Z}_{n}=\mathbf{Z} / n \mathbf{Z}$. Observe that if $(k, n)=1$, then the application "multiplication by $k$ " is an automorphism of the ring $\mathbf{Z}_{n}$, whence we easily deduce (by using (5)) that $\mu\left(A_{n}^{k}\right)=\mu\left(A_{n}\right)=s_{n}$. More generally, if $(k, n)=m, k=m a, n=m b$, then "multiplication by $k$ " ("multiplication by $a$ ") ("multiplication by $m$ "); the image of "multiplication by $m$ " is $m \mathbf{Z}_{n} \simeq \mathbf{Z}_{(n / m)}=\mathbf{Z}_{b}$, and "multiplication by $a$ " is an automorphism of the subring $m \mathbf{Z}_{n}$ because $(a, b)=1$. It follows from these observations, along with (4) and (5), that

$$
\mu\left(A_{n}^{k}\right)=\mu\left(A_{n}^{m}\right) \geqslant\left[\left(m \mu_{n}-1\right) / n\right]+1 \text { for all } k=1,2, \ldots
$$

Thus, in order to complete the proof, we only have to show that if $1<m<n$ and $m \mid n$, then $\mu\left(A_{n}^{m}\right)=\left[\left(m \mu_{n}-1\right) / n\right]+1$. By using (5), we have

$$
\begin{aligned}
& \mu\left(A_{n}^{m}\right)=\max _{0 \leqslant t<n}\left\{s _ { n } \left(\mathbf{c}\left\{j: j m \equiv t(\bmod n), 0 \leqslant j<a_{n}\right\}\right.\right. \\
&+\left(s_{n}-1\right)\left(\mathbf{c}\left\{j: j m \equiv t(\bmod n), a_{n} \leqslant j<n\right\}\right) \\
&= \max _{0 \leqslant t<n}\left\{s_{n} \cdot \mathbf{c}\{j: j m \equiv t(\bmod n)\}-\mathbf{c}\left\{j: j m \equiv t(\bmod n), a_{n} \leqslant j<n\right\}\right\} \\
&= s_{n} \cdot \mathbf{c}\{j: j m \equiv 0(\bmod n)\}-\mathbf{c}\left\{j: j m \equiv 0(\bmod n), a_{n} \leqslant j<n\right\} \\
&= m s_{n}-\mathbf{c}\left\{l: a_{n} \leqslant l(n / m)<n\right\} \quad\left(\text { using the fact that } \alpha_{0}=s_{n}, \alpha_{1}=s_{n},\right. \\
& \quad \alpha_{1}=s_{n}, \ldots, \alpha_{a_{n}-1}=s_{n}, \alpha_{a_{n}}=s_{n}-1, \ldots, \\
&= m s_{n}-\mathbf{c}\left\{l:(m / n) a_{n} \leqslant l<m\right\}=m s_{n}-\left[m-\left(m a_{n} / n\right)\right] \\
&=\left.m s_{n}-\left[m s_{n}-\left(m \mu_{n} / n\right)\right] \quad \text { insing }(6)\right) . \\
&= m\left[\left(\mu_{n}-1\right) / n\right]+m-\left[m\left[\left(\mu_{n}-1\right) / n\right]+m-\left(m \mu_{n} / n\right)\right] \quad \text { (using sequence) }
\end{aligned}
$$

Let $\mu_{n}=h n+g$, where $h \geqslant 0,0 \leqslant g<n$; then a straightforward computation shows that

$$
\mu\left(A_{n}^{m}\right)=\left\{\begin{array}{l}
m h \quad \text { if } g=0, \\
m h+m-[m-(m g / n)] \quad \text { if } 1 \leqslant g<n .
\end{array}\right.
$$

On the other hand,

$$
\left[\left(m \mu_{n}-1\right) / n\right]+1=\left\{\begin{array}{l}
m h \text { if } g=0, \\
m h+[(m g-1) / n]+1 \text { if } 1 \leqslant g<n .
\end{array}\right.
$$

Thus, $\mu\left(A_{n}^{m}\right)=\left[\left(m \mu_{n}-1\right) / n\right]+1=m h$ for the case when $g=0$. If $\ln / m<g \leqslant$ $(l+1)(n / m)$, then

$$
\mu\left(A_{n}^{m}\right)=\left[\left(m \mu_{n}-1\right) / n\right]=m h+l+1, \quad l=0,1,2, \ldots, m-1,
$$

whence we conclude that $\mu\left(A_{n}^{m}\right)=\left[\left(m \mu_{n}-1\right) / n\right]+1$ for all $m \mid n, 1<m<n$.

The proof of Lemma 3 is now complete. 
Proof of Theorem 1. Suppose that $\mathscr{H}$ is a Hilbert space with orthonormal basis $\left\{e_{i}\right\}_{i=1}^{\infty}$. Define

$$
\begin{aligned}
& A_{1}=1^{\left(\mu_{1}\right)} \quad \text { on } \bigvee\left\{e_{1}, e_{2}, \ldots, e_{\mu_{1}}\right\}, \\
& A_{2} \text { on } \bigvee\left\{e_{\mu_{1}+1}, e_{\mu_{1}+2}, \ldots, e_{\mu_{1}+\mu_{2}}\right\}, \\
& \quad \vdots \\
& A_{n} \text { on } \bigvee\left\{e_{\mu_{1}+\mu_{2}+\ldots+\mu_{n-1}+1}, e_{\mu_{1}+\mu_{2}+\ldots+\mu_{n-1}+2}, \ldots, e_{\mu_{1}+\mu_{2}+\ldots+\mu_{n}}\right\},
\end{aligned}
$$

exactly as in Lemma 3.

Let $\left\{r_{n}\right\}_{n=1}^{\infty}$ be any strictly decreasing sequence of positive reals with $r_{1}=1$. Now we define

$$
T(M)=\bigoplus_{n=1}^{\infty} r_{n} A_{n} \in \mathscr{L}(\mathscr{H}) .
$$

It is easy to check that $T(M)$ is normal, $\|T(M)\|=1$,

$$
\sigma(T(M)) \subset\left(\bigcup_{n=1}^{\infty}\left\{r_{n} e\left(\frac{j}{n}\right): j=0,1,2, \ldots, n-1\right\}\right)^{-},
$$

and

$$
\mu\left(T(M)^{k}\right)=\sup _{n} \mu\left(A_{n}^{k}\right) \quad(k=1,2, \ldots) .
$$

By using (2) and Lemma 3, we deduce that

$$
\mu\left(A_{n}^{k}\right)=\mu\left(A_{n}^{(k, n)}\right)=\left[\left((k, n) \mu_{n}-1\right) / n\right]+1 \leqslant \mu_{k}=\mu\left(A_{k}^{k}\right)
$$

for all $n=1,2, \ldots$, and therefore

$$
\mu\left(T(M)^{k}\right)=\mu_{k} \quad \text { for all } k=1,2, \ldots
$$

Furthermore, if $r_{n} \downarrow 0$ fast enough, then $T(M)$ is a nuclear operator. (It suffices to take $\left.r_{n}=\left(n+\mu_{n}\right)^{-4}, n=1,2, \ldots\right)$

This proves Theorem 1 for the case when $\mathscr{X}$ is a Hilbert space. If $\mathscr{X}$ is not a Hilbert space, then it is enough to repeat the above construction with the orthonormal basis replaced by a normalized Markushevich basis (see [2]; the details are left to the reader).

Proof of THEOREM 2. We begin by constructing a nuclear normal operator $T(M)$ exactly as in the previous proof.

Let $L$ be a diagonal normal operator defined by $L f_{i j}=t_{i} e\left(v_{j}\right) f_{i j}$ with respect to an orthonormal basis $\left\{f_{i j}\right\}_{i j=1}^{\infty}$, where $\left\{t_{i}\right\}_{i=1}^{\infty}$ is a denumerable dense subset of (distinct points of) $(0,1) \backslash\left\{r_{n}\right\}_{n=1}^{\infty}$ and $\left\{e\left(v_{j}\right)\right\}_{j=1}^{\infty}$ is a denumerable dense subset of the unit circle such that $v_{j}$ and $v_{j} / v_{h}$ are irrational for all $j$ and, respectively, for all $h \neq j$. Then $L$ is a normal operator, the set of all eigenvalues of $L^{k}$ is disjoint from the set of all eigenvalues of $T(M)^{k}$ for each $k=1,2, \ldots$, and it straightforward to check that

$$
\begin{aligned}
\mu\left(\{T(M) \oplus L\}^{k}\right) & =\max \left\{\mu\left(T(M)^{k}\right), \mu\left(L^{k}\right)\right\} \\
& =\max \left\{\mu_{k}, 1\right\}=\mu_{k} \text { for all } k=1,2, \ldots
\end{aligned}
$$


(see [1]), and

$$
\sigma(T(M) \oplus L)=\sigma(L)=\{\lambda:|\lambda| \leqslant 1\} .
$$

Thus, the normal operator $N(M)=T(M) \oplus L$ satisfies all our requirements.

\section{REFERENCES}

1. J. Bram, Subnormal operators, Duke Math. J. 22 (1955), 75-94.

2. J. T. Marti, Introduction to the theory of hases, Springer-Verlag, Berlin, Heidelberg and New York, 1969.

Department of Mathematics, Arizona State University, Tempe, Arizona 85287 